Conclusion Telemedicine had been shown to be a safe and effective method in the care of stable cardiology patients. The social, economic and environmental benefit of tele-consultation raised exciting opportunity for future service designs. Collaboration between hospital specialist, management team, community health providers and communication service suppliers are vital to ensure the sustainable implementation of telemedicine in cardiology.

Conflict of Interest none

\section{Cardiac rhythm management}

\section{AUTONOMIC AND VASCULAR FUNCTION CHARACTERISTICS IN PATIENTS WITH ATRIAL HIGH RATE EPISODES}

${ }^{1}$ Ahsan Khan, ${ }^{2}$ Reem Alsharari, ${ }^{3}$ Rehan Junejo, ${ }^{4}$ Neil Thomas, ${ }^{5}$ James Fisher, ${ }^{6} \mathrm{G}$. Lip. ${ }^{1}$ University of Birmingham, Birmingham, UK; ${ }^{2}$ Institute of Cardiovascular Sciences, University of Birmingham; ${ }^{3}$ Manchester Metropolitan University; ${ }^{4}$ Institute of Applied Health Research, University of Birmingham; ${ }^{5}$ University of Auckland; ${ }^{6}$ Liverpool Centre for Cardiovascular Science, University of Liverpool and Liverpool Heart \& Chest

\subsection{6/heartjnl-2021-BCS.83}

Introduction Patients who experience frequent atrial high rate episodes (AHREs), recorded on implantable cardiac devices, are at an increased risk of stroke and systemic embolism. Increased duration of AHRE is related to higher risk of thromboembolic event. The underlying pathophysiology is unclear; however, autonomic and/or endothelial dysfunction may contribute. We tested the hypothesis that patients with high AHRE burden have worse autonomic and

\begin{tabular}{|c|c|c|c|}
\hline & $\begin{array}{l}\text { Low AHRE group } \\
(\mathrm{n}=22)\end{array}$ & $\begin{array}{l}\text { High AHRE group } \\
(n=22)\end{array}$ & $\mathbf{P}$ \\
\hline Age, years & $74.5[67.5-81]$ & $72[60.8-82.8]$ & 0.56 \\
\hline Sex (males/females) & $15 / 7$ & $19 / 3$ & 0.15 \\
\hline CHA2DS2-VASC score & $3 \pm 2$ & $3 \pm 2$ & 0.93 \\
\hline BMI $\left(\mathrm{kg} / \mathrm{m}^{2}\right)$ & $29.6[26.9-32.9]$ & $27.7[24.8-36.7]$ & 0.70 \\
\hline Heart rate (bpm) & $69[61-75]$ & $68[60-80]$ & 0.97 \\
\hline Systolic BP (mm/Hg) & $152 \pm 18$ & $135 \pm 17$ & 0.003 \\
\hline Ejection fraction (\%) & $59[55-62]$ & $55[40-58]$ & 0.009 \\
\hline Baseline diameter (mm) & $4.6[4.3-5.0]^{\mathrm{a}}$ & $4.8[4.5-5.2]^{\mathrm{a}}$ & 0.36 \\
\hline Absolute FMD change (mm) & $0.2[0.2-0.4]^{\mathrm{b}}$ & $0.1[0.1-0.3]^{\mathrm{b}}$ & 0.07 \\
\hline FMD (\%) & $5.5[3.4-7.6]^{\mathrm{b}}$ & $3.1[1.9-6.2]^{\mathrm{b}}$ & 0.04 \\
\hline Shear rate stimulus [sec.-1] & $5592[4174-9815]^{b}$ & $4173[2458-10547]^{b}$ & 0.41 \\
\hline SDNN (ms) & $41[28-55]^{\mathrm{a}}$ & $60[39-80]^{a}$ & 0.12 \\
\hline rMSSD (ms) & $40[27-54]^{\mathrm{a}}$ & $58[39-77]^{\mathrm{a}}$ & 0.10 \\
\hline pNN50 (\%) & $5[2-23]^{b}$ & $17[2-60]^{b}$ & 0.12 \\
\hline $\operatorname{LF}\left(m s^{2}\right)$ & $575[137-1013]^{\mathrm{a}}$ & $665[-264-1593]^{\mathrm{a}}$ & 0.83 \\
\hline $\mathrm{HF}\left(\mathrm{ms}^{2}\right)$ & $160[66-687]^{b}$ & $112[42-1839]^{b}$ & 0.98 \\
\hline LF normalised/HF normalised & $0.8[0.5-1.0]^{b}$ & $0.9[0.5-1.4]^{b}$ & 0.84 \\
\hline SD1 (ms) & $29[19-38]^{a}$ & $41[28-54]^{\mathrm{a}}$ & 0.11 \\
\hline $\mathrm{SD} 2$ (ms) & $49[31-67]^{a}$ & $67[47-87]^{a}$ & 0.15 \\
\hline
\end{tabular}

Descriptive data are presented as numbers (withpercentages). Normallydistributed data are expressed as mean $[95 \%$ confidence intervals (CI)]. Identifiedby superscript a. Non-normally distributed data are displayed as median $[95 \% \mathrm{Cl}]$. Identified by superscript b. Statistical differences were tested usingindependent t-test (for parametric data) or Mann-Whitney $\mathrm{U}$ test (for non-parametricdata). Significancep $\mathrm{f} 0.05$ vascular function compared to patients with low AHRE burden.

Methods We studied 44 patients split in two groups: high AHRE burden (longest AHRE $\geq 24$ hours; $n=22$ ) and low AHRE burden (longest AHRE $<24$ hours; $n=22$ ). Resting time- and frequency-domain measures of HRV were determined to index cardiac autonomic function. High-resolution ultrasound was used to measure brachial artery diameter at rest and during reactive hyperaemia (endothelium-dependent flow mediated dilation (FMD)).

Results FMD was higher in the low AHRE burden group compared to high AHRE group (5.5\% [95\% confidence interval (CI):3.4-7.6] vs 3.1\% [95\% CI:1.9-6.2]; p=0.04) (table $1)$. Mean heart rate $(p<0.001)$ and AHRE burden $(p=0.02)$ were independent predictors of FMD on multivariate analysis. Time-domain, frequency-domain and non-linear indices of HRV were not significantly different between the groups. BMI was an independent predictor of HRV on stepwise multivariate analysis $(p=0.002)$.

Conclusion Endothelium-dependent FMD is impaired in patients with high AHRE burden, while HRV derived indices of autonomic function were not affected by AHRE burden. Endothelial dysfunction may play an important role in the adverse outcomes seen in patients who experience frequent AHRE.

Conflict of Interest None

\section{IMPACT OF THE INTRODUCTION OF AN INTEGRATED MULTI-PROFESSIONAL PATHWAY FOR ATRIAL FIBRILLATION ON LONG-TERM OUTCOMES}

Saadia Aslam, Clare Vickers, Wendy Veevers, Layla Farrar, Elizabeth Hartley, Karthik Viswanathan. Calderdale and Huddersfield NHS Foundation Trust, Halifax, UK

\subsection{6/heartjnl-2021-BCS.84}

Background An integrated structured approach to atrial fibrillation (AF) management is recommended by ESC. ${ }^{1}$ However, limited data is available on its 'real-world' impact on anticoagulation uptake and subsequent AF-related hospitalisation and stroke rates.

Purpose To evaluate the long-term impact of the introduction of a community-wide integrated AF service based in a secondary care hospital.

Methods In September 2017 we implemented a new integrated pathway for patients with newly diagnosed 'symptomatic' atrial arrhythmias across two regions (population 450,000) in England in collaboration with primary care. All patients were seen in a one-stop multi-professional clinic (Arrhythmia nurse and Electrophysiologist with ECG and echocardiogram) within 2 weeks. They underwent standardised screening for modifiable risk factors, counselling regarding diagnosis/ anticoagulation and received an individualised AF management plan as well as access to a nurse-led telephone helpline. We followed up the first 126 consecutive patients for a period of 12 months.

Results Baseline characteristics are shown in table 1, showing a high incidence of modifiable risk factors (previously un-identified in the majority). After review in clinic, 30-day AFrelated readmission rates to hospital were low $(1.6 \%, n=2)$ as compared to historical local data $(5.8 \%)$. This was sustained at 3 months $(4.7 \%, \mathrm{n}=6)$ and at 12 months $(7.9 \%, \mathrm{n}=10)$. $99 \%$ of 83 of eligible patients (CHA2DS2-VASc score of $>2$ 